\title{
The Effect of Reinforcement and Punishment on Employee Performance
}

\author{
ABM Asadullah ${ }^{*}$, Nurita Binti Juhdi², Md Nazrul Islam ${ }^{3}$, Alim Al Ayub Ahmed ${ }^{4}$, \\ ABM Abdullah ${ }^{5}$ \\ ${ }^{1}$ Department of Economics and Management Sciences, International Islamic University Malaysia, Kuala \\ Lumpur, MALAYSIA \\ ${ }^{2}$ Assistant Professor, Department of Economics and Management Sciences, International Islamic \\ University Malaysia, Kuala Lumpur, MALAYSIA \\ ${ }^{3}$ Graduate School of Management, International Islamic University Malaysia, Kuala Lumpur, MALAYSIA \\ ${ }^{5}$ Visiting Full Professor, Accounting School, Jiujiang University, Jiujiang, Jiangxi, CHINA \\ ${ }^{4}$ Assistant Coordinator, Sajida Foundation, BANGLADESH \\ *Corresponding Contact: \\ Email: asadullah.iium@gmail.com
}

Manuscript Received: 30 March 2019 - Revised: 15 June 2019 - Accepted: 05 July 2019

\begin{abstract}
There is a direct reciprocal relationship between the employee and the organization. The employee behavior is very important for any organization whose culture greatly influences the former. This study examines the behavioral theories and modern theories that aim at motivating the employee to increase his performance by reinforcements and punishments. Based on the study, an attempt has been made to deeply explore the internal and external factors influencing employee's behavior. Firstly a case study of external factors on employee behavior has been studied considering the behavioral theory. Secondly, a case study of internal factors on employee behavior have been studied considering modern theory and rejecting the behavioral theory. The results of this investigation from both the cases portray that reinforcement and punishment significantly influence motivation, values, decision making, tackling conflicts and overall performance of the employee.
\end{abstract}

Keywords: reinforcement, punishment, cognitive, behavior

This article is is licensed under a Creative Commons Attribution-NonCommercial 4.0 International License.

Attribution-NonCommercial (CC BY-NC) license lets others remix, tweak, and build upon work non-commercially, and

although the new works must also acknowledge \& be non-commercial.

\section{INTRODUCTION}

To be combative and sustainable in today's fluctuating market, organizations constantly pressurize employees to deliver high performance (Sitkin et al., 2011). Organizational culture and employee behavior have been stipulated by a vast number of theorists and psychologist. The behavior is either the influence of inner emotions or the outcome of your environment. The most appealing as far as the external effect on behavior had been Skinner, who has experimentally studied on rats, pigeons and even human behavior (Skinner, 2014). Nowadays, an employee in an organization must face an extremely 
diverse environment with a high level of complexity and uncertainty. If employee aims to survive in such a dynamic environment, they must develop capabilities and attitude to sense these changes and to offer accurate responses to them, gaining new deals and competitive advantages to utilize (Meglino and Ravlin, 1998). In the organizational culture, employees share a common value and ethics. This helps in developing interpersonal relationships, a relaxed working environment with a sense of responsibility leading to the overall goal of the organization. Behavior control has been defined as an organization's capability to sense environmental changes and to respond efficiently and effectively (Conger and Kanungo, 1988). Simultaneously, organizations in today's scenario are required to become more respectful of their employee. Thus a step by step development towards internal culture between the organization and employ based on behavior, values, and a set of beliefs is defined by (Heskett and Kotter, 1992). This implies that in today's world sustainability are both considered as performance indicators for modern industries (Otley, 1994). Moreover, several studies have shown that employees' behavior exerts a high impact on industrial growth (Griffin et al., 2007). The main focus is on the relation between behavior and performance (Wermers, 2003). The previous literature has developed few attempts to study the effects of employee behavior on the performance of organizations but the effect of external and internal environment in the development of behavior and consequence that controls it need to be studied independently. We cannot deny the inner environment whether emotions or mind or believes that too play an important part in shaping the behavior. Source of behavior is described as the external environment but is dismissed by cognitive scientists developing intricate internal information processing models and concluded that its methods are irrelevant to studying how animals and persons behave in their natural and social environment. We in this paper go deeper into classical behaviorist theories as an independent case study, giving us huge historical data on external factors affecting behavior. Another independent case study highlighted in this paper is a cognitive scientist who rejects the behaviorist theory due to the assumption of humans as a machine with no inner effects. We after comparison of both theories accept the ideology of behaviorist with some modification as external environment has worked very well on rats, pigeons, and even humans. At the same time, we see the effect of the inner environment on the employee's performance suggested by the cognitive approach and accept human emotions and feeling. Also, emphases have been laid out in developing mentality positive, loyal, sincere, motivational and accountable employee. In the author's view, this combination of external and internal behavior could thrive an employee physically, spiritually and mentally towards the upliftment of the organization but these effects for better results should be studied individually.

\section{A Case Study on Behaviorism}

Psychology research didn't originate from the vacuum. It depends on three primary columns. One critical column is logic, another column is a medicine which discusses the mental process and the third column is science. Psychophysics, functionalism, structuralism, and behaviorism all added to the improvement of comprehension. One of the significant school of psychological science that influenced subjective hypothesis has been behaviorism. A portion of the designers of the social hypothesis is Ivan Pavlov, Skinner, Watson as specified in the literature above. Analyst experimented on certain creatures such as human, rats, pigeons etc. and found the propensity of trouble making. They didn't act the way the therapist expected them to act. There is numerous hypothetical case of mischief. Behavior hypothesis inability to precisely anticipate complex intuitive behavior. 
Newborn child connection hypothesis recommends that youngsters who are even more safely joined to guardians at an early stage in life are generally free at later stages. The learning is a long-haul change in the behavior that depends on understanding. Behavior hypothesis has two methodologies. Firstly, the observable and quantifiable methodology known as a psychological hypothesis which has been deeply investigated in this study. Secondly, the mental and passionate methodology known as a psycho-scientific hypothesis which too has been highlighted in this study. Below shown in fig.1 is the Skinner model of behaviorism considering human as a being influential only to external environment.

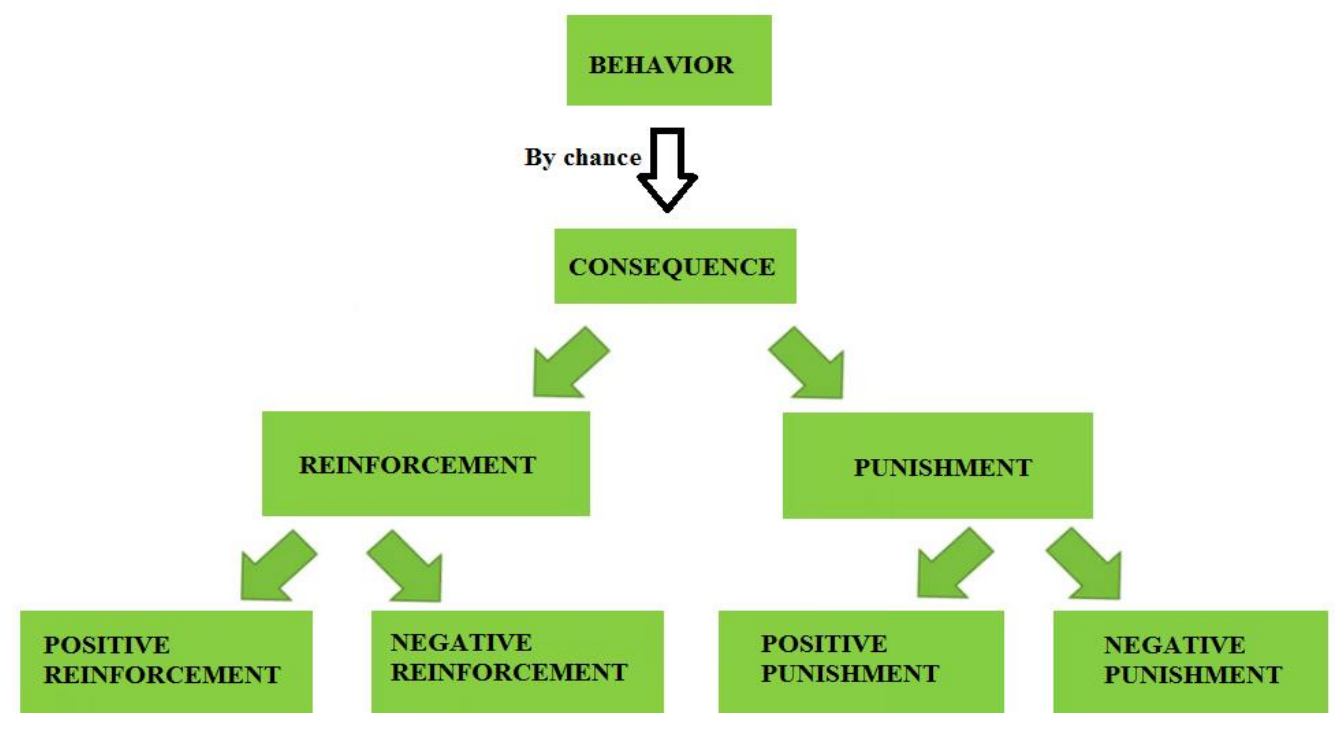

Figure 1: Skinner Model

\section{Theory of Watson}

He is the founder of behaviorism and accepts just what you can gauge. External factors figure out our identity. Behaviorism is identified with action. Watson examined behaviorism by two analyses i.e. "Little Albert" and "Kerplunk analyze". Little Albert explore was done on an 11-month kid with a white rat. At the point when youngster approaches rat, an uncommon loathsomeness tone rang which made tyke dread of the rat. Behaviorism incorporates dread idea instead of dread. Such introductions are reinforcement, such lights are upgrades, such lever pressings are reactions, and such affiliations are learning histories. His work was on the traditional molding of behavior. He contended that given dozen sound newborn children to bring them up in his natural condition could promise them to be a doctor, an engineer and even hoodlum (Watson, 1920). He had faith in external impacts which could be estimated and well measured.

\section{Theory of Tolman}

He worked on sign learning and trusted that creatures were obtaining learning about their surroundings called insights. He found that rats being transported around his research facility were demonstrating proof that they had picked up something about the space in later tests. He says we sometimes learn without boost and support and gave the hypothesis of purposive behavior. In his book (Tolman, 1951), purposive behavior of creature and men, he explored the psychological hypothesis of learning. As indicated by 
this hypothesis, a few people don't display their scholarly learning. Be that as it may, when it is required or when they get support, they demonstrate their insight like others.

\section{Theory of Pavlov}

Russian Psychologist named (Pavlov, 1941) was the pioneer of classical conditioning. He demonstrated nourishment the canine and rang the ringer in the meantime. They took in the ringer is a call for sustenance and responded on it. In the long run, they figured out how to expect nourishment at the sound of a chime. We call the sustenance unconditioned improvement and call mutts to salivate as an unconditioned reaction. This is the very same way how it functions for people. Outside impacts explain some behavior of automatic passionate and physiological reactions. So, he concluded that humans learn to associate a stimulus with a response and after some time our body does it automatically in the presence of the stimulus. Any stimulus in an organism can perceive and can listen to any reaction that the organism can make. This means that smell, sound or sight can influence our muscles to become tense or relaxed and make our moods fluctuate or even are attitude is formed. Here our response is involuntary. Expelling a positive reinforcement given to an ominous behavior, in the long run, evacuate the conduct. Established molding can confront annihilation where the behavior is befuddling. This happens when in Pavlov's examination the ringer is ringing however the dog quits getting food. Thus, if employees are stopped giving reinforcement such as increment for their work then it might lead to an adverse effect.

\section{Theory of Skinner}

All people are machines and controlled by the result. Skinner discussed operant molding i.e. results that prompt changes in deliberate conduct. He trusted that ethical state of mind in the public arena are altogether formed by outer components. Individuals who carry on good, feel moral at the same time. Social authorizations are the motivation behind why individuals feel moral and carry on ethically. He additionally separated it in to support and discipline and ignored unrestrained choice. All behavior starts as oblivious conduct. Positive means expansion of an improvement. Negative means the expulsion of an upgrade. As per this hypothesis, natural outcomes are intense instruments that supervisors and can use to shape behavior. Skinner saw that either positive or negative practices can influence the employees' behaviour. As per this hypothesis, natural outcomes are intense instruments that supervisors and can use to shape behavior. Skinner saw that either positive or negative practices can be focused on, however in a business setting, concentrating on remunerating wanted conduct enables employees to create positive propensities and is less inclined to encourage hatred than a more reformatory methodology.

\section{A Case Study on Cognition Theory}

\section{Theory of Chomsky}

Chomsky condemned behavior model not to be right and standard science. He clarifies that beings depend on hereditary programming and improvement of dialect resembles a fetus. Why chimpanzee can't create like human behavior? For what reason, wouldn't they be able to talk like humans? There is some inner hereditary property that recognizes humans from different animals. This is same about dialect. Why this mystery and move in mind. Chomsky has been one of behaviorism's best and harming pundits. Chomsky charged that behaviorist models of dialect learning can't clarify different realities about dialect securing. A kid's semantic capacities give off an impression of being fundamentally 
under-dictated by the proof of verbal conduct offered to the tyke in the brief time frame in which he or she procures those capacities. By the age of four or five youngsters have a relatively boundless ability to comprehend and deliver sentences which they have never heard. The issue to which Chomsky alludes, or, in other words of conduct limits exceeding individual learning narratives, appears to go past only the issue of etymological conduct in youthful youngsters. It has all the earmarks of being a major truth about people that our sensitivities and social limits regularly outperform the constraints of our individual learning chronicles. Our history of reinforcement regularly is excessively ruined, making it impossible to decide remarkably our behavior. Much adapting, subsequently, appears to require prior or intrinsic authentic structures inside which learning happens (Cowie, 1998). Skinner didn't examine mind as you couldn't straightforwardly see it however what you could see is behavior and planned skinner boxes to quantify reactions considering support. Likewise, discussions of semantics among Chomsky and Skinner, opened a space for various relationship how the brain works. These theories lead to the development of cognitive approach and was a turning point in various disciplines including philosophy and psychology. Further computers development gave a pathway to modern cognitive approach towards human behavior. Chomsky book syntactic structure talks about linguistics but does give a new approach that human thoughts depend on inner environment as the computer. The data preparing dependent on successive stages to special process and each stage takes a shot at its own arrangement of inner portrayals Example (McLeod et al., 1998) in their book a prologue to associations displaying of cognatic forms on organic standards of the cerebrum, neurons gather data. They at that point passed data about the level of their information whether the edge had been come to or not. Mind structure tends to be layered. The impact of one neuron on another relies upon the quality of the association between them. Learning is accomplished by changing the qualities of the associations between neurons. The computers fill in as pleasant illustration. It could learn, store, control and had memory. It had interior segments and procedures. Most stream graphs were utilized for mind mapping. Today we have propelled our reasoning about our relationship not just about outside computers but rather the kind of inner programming that is covered up. The effect of innovation on the ascent of intellectual brain research. Computers representation data handling and mind similitude to connectionism. Make an interpretation of approaching data into various configurations. Ability to store projects, guidelines, and information. Computers can do mistake free counts. This made to the development of cognition psychology as it was quite clear that inner environment too effect the employee's behavior.

\section{Theory of Cognitive Scientist}

Behaviorism is rejected by a cognitive scientist who is subjective researchers creating mind-boggling inner data handling models and reasoned that its techniques are insignificant to concentrate how creatures and people carry on in their common and social condition. It is dismissed by neuroscientists beyond any doubt that the immediate investigation of the mind is the best way to comprehend the reasons for conduct. The greatest downside for behaviorism's downfall is its responsibility to the proposal that conduct can be disclosed without reference to mental action. Numerous logicians and analysts discover this postulation pitifully prohibitive. They dismiss behaviorism because of it. The basic inquiry is the thing that considers a record of a reinforcement history. Numerous analysts give up all hope of portraying what considers a record without hypothesizing interior memory states and inner handling over those states (Roediger et al., 1998). Since memory states serve to record past encounters and fortifications and serve 
referentially to remain for improved conditions which the life form recollects that, they are usually portrayed as inner mental portrayals. One characterizing highlight of conventional behaviorism is that it endeavored to free brain science from conjecturing about how creatures and people speak to their condition. This was vital, verifiable because it appeared that behavior and condition associations are a ton clearer and more sensible tentatively than inside portrayals. The thinker analyst (Place, 2000), albeit generally thoughtful to uses of behaviorism to issues of the psyche, contended that qualia can't be dissected in behaviorist terms. Indeed, it is enticing to hypothesize that feels influence non-subjective components of interior handling, and that they, for instance, add to the excitement, consideration, and receptivity to affiliated molding. This gave rise to the social learning hypothesis. An arrangement of adapting most generally connected with behaviorist Albert Bandura (Bandura, 1976), is most usually connected in instructive settings. First is model behavior for representatives. The initial phase in encouraging a behavior in the social learning hypothesis is displaying that conduct. Rather than basically advising employees what they need them to do, entrepreneurs should physically display the behavior, showcasing the procedure they trust those under them will pursue. By observing the behavior demonstrated, representatives can build up a more characterized comprehension of what the behavior involves and encounter more accomplishment in completing the behavior being referred to. Secondly, encourage the representatives to mimic your practices. In the wake of demonstrating, top managers ought to verbally support behavior impersonation. They can do this in two different ways. To begin with, they can specifically tell the worker that they need him to do as he saw demonstrated. Also, they can remunerate representatives who pursue the demonstrated conduct in an open form, enabling different laborers to see that this conduct is the one that they need all specialists to embrace. Thirdly observe the representative as he completes the conduct. Entrepreneurs should watch out for specialists, ensuring that they are following the strategies they demonstrated. On the off chance that the workers are observed not to pursue this methodology, the pioneer should venture in and remedy the representative's conduct, urging him to adjust his behavior. Finally, establish ramifications for behavior deviation. At the point when representatives neglect to pursue the practices that the pioneers are endeavoring to embrace, there must be ramifications for doing as such. These results may include basic verbal remedy or be as serious as formal authorizations.

\section{Results and Discussion}

\section{Skinner Indicator}

Skinner stated, the annihilation of undesired behavior results from the nonappearance of encouraging feedback, not from discipline. This implies offering an impetus when work surpasses desires, encouraging feedback, and concentrating on elimination by withholding it or withholding extra benefits when targets are not met. For instance, you may offer a reward for deals in abundance of your week by week focus on, a long lunch for meeting the objective and a standard lunch, withholding both the reward and the long lunch, for neglecting to meet it. An unmistakable outline in this regard is being highlighted in his book Skinner's Beyond Freedom and Dignity. In his book about behavior examination he albeit basically sees western philosophy, moral qualities and being human. He tentatively researched fortification and discipline. As indicated by Skinner, Great and Awful, devout and corrupt are equivalent words utilized relying upon the association. Skinner weights on outcomes as the key to controlling human behavior. Results can be a reward, subsequently controlling behavior by outer factors however cognizant or oblivious powers 
can't be seen or estimated, so can't control behavior by hereditary components. In this manner, four noteworthy behavior move by result has been contemplated. Encouraging feedback is a behavior which drives you to reward and in this way, you will probably rehash such behavior. Negative support is a behavior that outcomes in the expulsion of something unpalatable, and hence you will probably rehash such behavior. Positive punishment is a conduct including expansion of repulsive upgrade. Negative punishment is a behavior including the evacuation of wonderful improvement. A section from Skinner's model, this paper suggests that the effect of the diverse factors on behavior can significantly impact the efficiency and execution of organization. Innovation power at industry level ought not just to control employee behavior by remunerations and punishment, however others factors, for example, versatility to change, learning based work and decentralization of power, among others ought to likewise be considered. Just external impacts and not interior considerations and feeling shift behaviour and can be very useful for an organization to mold and control employee's behavior.

\section{Work Environment Behavior}

It is formed by four speculations: positive and negative support and positive and negative punishment. Positive and negative reinforcement are both used to make positive conduct. You must examine a circumstance and figure out which kind of reinforcement would be best. One approach to take a gander at it is to treat uplifting feedback like an objective and negative support as an update. Negative support winds up power when the representative is helped to remember the negative movement that was evacuated to create a positive outcome. The thoughts introduced could be additionally created contemporary difficulties in comprehension for future human living prospects. What practices will be most vital for accomplishing our technique? Encouraging feedback includes remunerating a representative for completing a great job. A model would give a representative a paid getaway day for surpassing month to month creation objectives. Antagonistic support includes evacuating something bothersome to change conduct - for instance, suspending everyday gatherings with an administrator to talk about occupation execution after a worker has raised his own generation levels. Utilizing positive and negative fortification in the work environment ought to be done just when painstakingly observing worker response to guarantee the proposed results. The intensity of acclaim in the work environment can move representatives from indifference or hatred to bliss and efficiency. Uplifting feedback makes individuals feel acknowledged and supported, which can be inspiring and fulfilling. Knowing the pluses and minuses can enable you to actualize a compelling encouraging feedback program that boosts efficiency and execution at your working environment. Rewards fiscally or non-monetarily assume a crucial job in propelling representatives to fill in as compelling and effective work drive. Positive and negative support is normal devices in association culture to rouse specialists. An uplifting feedback is a reward or motivation offered to a worker for meeting certain execution principles. A negative reinforcement is the utilization of an outcome, for example, lost pay or a downgrade, to dishearten a worker from failing to meet expectations or carrying on upsettingly or unscrupulously. Bosses utilize guide support to manage their specialists to perform wanted assignments and to keep away from undesirable practices. While most generally connected with altering youngsters' practices, fortification, both positive and negative, is likewise a viable method to change the conduct of grown-ups and is more typical in the work environment than people may at first speculate. 


\section{Work Environment Reward}

It is uplifting feedback that fills in as a method for empowering representatives as a positive work compel and to meet a specific level of desire. Uplifting feedback at work happens when a man is remunerated to empower a behavior. Verbal support gives representative gesture of congratulations that energizes him toward being the most ideal specialist, written affirmed acclaims gives feeling of part to a major family, employee affirmation, for example, worker of-the-month, representative of-the-year and so forth tries workers certain that their endeavors don't go unnoticed, tangible rewards, for example, organization logo journal, pen, gift and so forth propels representatives, pay climb will summon a coveted reaction. So, clear and reasonable expectations are set. The use of reinforcement to motivate employees should be a positive experience. Indistinct undertaking desires and assessment guidelines baffle employees and decrease the inclination to endeavor the coveted behavior. Envision your reaction to an audit in which you were advised to improve the situation with no subtle elements. Identify Strong Motivators Working with representatives to recognize customized inspirations, or reinforcement is destined to create the coveted outcomes. Rousing prizes are basic to the achievement of your program since they must entice enough for representatives to work to change their behavior. Encouraging Desirable Behaviors. Most managers need to energize positive representative conduct, for example, timeliness, solid cooperation and quality generation. As per reinforcement theory, picking one positive ascribe to focus at once and applying encouraging feedback strategies with an attention on termination of the negative behavior, can enable you to transform alluring characteristics into solid work propensities after some time.

\section{Work Environment Sanctions}

It is negative reinforcement that fills in as a method for demoralizing representatives from participating in taboo exercises. Operant conditioning is a behavior hypothesis that proposes conduct is most effectively altered when it creates a negative result. This hypothesis can be valuable when connected to the work environment in a few different ways, from tending to how representatives associate with each other and with customers to how fiscally fruitful an association is yearly. Unmistakably conveying desires and negative outcomes of poor choices and practices in advance are basic to a compelling administration style. Organization strategy manuals and lead codes, for example, often recognize principles of worthy behavior and order forms on the off chance that they aren't met. The fact of the matter is to dishearten workers from bothersome results and toward positive prizes. Negative reinforcement combined with remunerations for positive practices makes a decent equalization. Negative support is some of the time mistook for discipline, yet in the working environment, negative fortification is a more compelling device for propelling representative conduct. Negative support urges representatives to perform better so they can have an obnoxious condition expelled from their workplace. It is one sort of motivational conduct characterized by Skinner as operant conditioning, alongside uplifting feedback, discipline, and termination. Constantly being reminded to be more productive, often seen as nagging or badgering by employees, is a negative reinforcement technique, workplace drug testing strives to create the desired environment through negative reinforcement. 


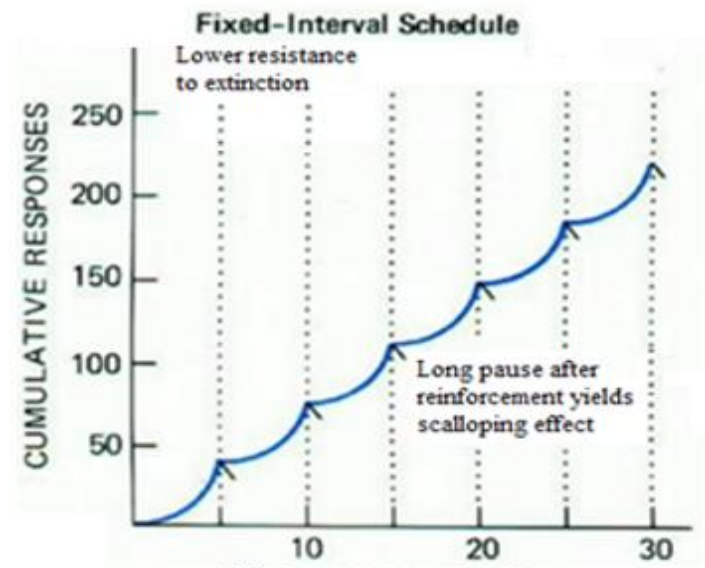

(a) TIME(MINUTES)

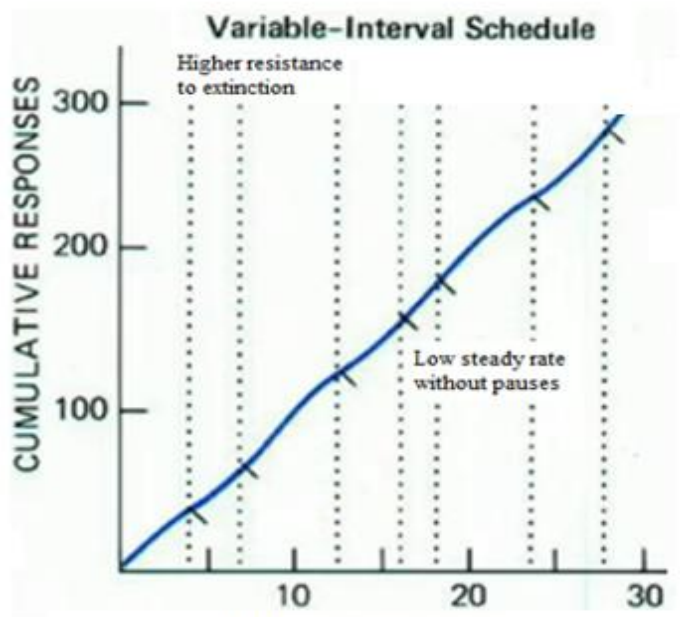

(b) TIME(MINUTES)

Figure 2: (a) Skinner Fixed and (b) Variable Interval Schedule

Consider a situation where employees who make mistakes are reprimanded in front of their peers or even spoken to harshly in private, concerns about negative reinforcement often refer to extreme use. Over-reliance on negative reinforcement, including punishments with no intent to correct behavior, leads to a low morale workplace characterized by anxiety and fear. Some managers overuse threats to drive employees to perform up to par. While this may lead to short-term production, it rarely contributes to strong, long-term success. Skinner outer impact plans conduct and human could be believed to be ear organic creature or machine. Everything we do is not remunerated and the same way all evil deeds are not rebuffed. Support remotely could decrease inborn inspiration among utilizes. Control bunch is less inventive and open. Behaviorism works for recognizable conduct. Behaviorism represents learning through individual involvement with the earth. Making the negative activity less desirable can help combat complacency with negative reinforcement. The most effective reinforcement schedule is a continuous schedule in which the reinforcement action occurs after every behavior. Intermittent reinforcement schedules can either be fixed or variable. A variable reinforcement can be along the lines of a 
job promotion. The more effective schedule is a variable one because employees know that the event will happen but when is not known. The effect is sustained behavior modification in anticipation of the event, positive and negative reinforcement are both used to create positive behavior. You need to analyze a situation and determine which sort of reinforcement would be most effective. One way to look at it is to treat positive reinforcement like a goal and negative reinforcement as a reminder.

\section{Both Positive and Negative Reinforcement}

A model of learning that incorporates both negative and positive feedback by finding a gap between them. Thus, improving cognitive functioning of employees. Sometimes the same act can include both negative and positive reinforcement. On one hand, this is positive reinforcement because the employees gain something they want when they reach your productivity goals. On the other hand, it is negative reinforcement because it involves escaping the noise and distraction -- a negative condition -- that motivates the employee productivity, one of the potential negative impacts of the two kinds of molding is carelessness.

\section{Work Environment Ethics and Conduct}

It is a spine for creating association culture and this depends on social learning hypothesis (Pavlov, 1941; McLeod et al., 1998) which models the behavior per necessity. Work tasks ought to be physically appeared by experienced representatives, work ethic from best progressive system can have broad consequences for the healthy organizational culture. Rewards are used to reinforce the behaviour you define and punishments are used to prevent the undesirable behaviour of employees. Extinction is a mean to prevent employee from performing a learned behaviour i.e. operant conditioning. Thus, the performance of employee will lead to following effects such as increase in profitability, employee's responsibility, global work of social decent variety, effective client benefit, sustained undertaking focused deals, high execution, positive workplace, positive venture results, maintain high worker assurance, tolerate pressure, work for extensive stretch, builds certainty, pleasurable experience, avoid negative impacts, for example, despondency, outrage and so forth.

\section{CONCLUSION}

This research highlights the external and internal factors to be very effective in enhancing employee's performance. The reinforcement and punishment come from behaviorist and cognitive approach and can physically, spiritually and mentally mold an employ as per organizational culture. First, it inspires the employee to move forward through encouragement and pull him back through threats. The reinforcement that one receives for a good act is due to some extraordinary field which might come from inner self or might come from outside environment. Likewise, punishment for ill effects is not a natural and unalterable consequence of man's acts. Some control is fully authorizing either from inside or outside to punish for his ills as well as to forget and forgive him. This is perhaps the strongest motivation and the most effective incentive for the employee to consider himself in no control or full control. We have highlighted the behavioral theories for external factors and found Skinners work of reward and punishment the most effective to shape humans but found the theory with a lot of flaws. Secondly, with the addition of cognitive approach the paradox of learning can be removed but motivate employees for a far productive and performance-driven organization. Cognitive processes are thoughts, perceptions, feelings, memories also influence our behavior. Unlike Skinner, cognitive 
psychologist explain clearly as to how are brain works, how are mind work and we understand our mind. Thirdly, the idea that punishment and reward are effective ways of controlling behavior can fail because neither all good deeds are rewarded, nor all bad deeds are punished. This paradox can only be understood, if science with some assumptions, were to accept the theory of religious belief system. Even if one may have to face some hardships and difficulties in the temporary life of this world, he would surely and strongly face it as his rewards are never ending and refutes the idea of Elliott (Sober, 2014). On the other hand, the wrongdoer will fate eternal punishment, no matter how rich and luxurious a life he may have led in this world. This proves a strong deterrent against an immoral and impious life.

\section{REFERENCES}

Bandura, A. (1976) "Self-reinforcement: Theoretical and methodological considerations," Behaviorism, vol. 4, pp. 135-155.

Conger, J.A. and Kanungo, R.N. (1988) "The empowerment process: Integrating theory and practice," Academy of management review, vol. 13, pp. 471-482.

Cowie, F. (1998) What's within?: nativism reconsidered: Oxford University Press.

Griffin, M.A.; Neal, A. and Parker, S.K. (2007) "A new model of work role performance: Positive behavior in uncertain and interdependent contexts," Academy of management journal, vol. 50, pp. 327-347.

Heskett, J. L. and Kotter, J.P. (1992) "Corporate culture and performance," Business Review. Vol, vol. 2, pp. 83-93.

McLeod, P.; Plunkett, K. and Rolls, E.T. (1998) Introduction to connectionist modelling of cognitive processes: Oxford University Press.

Meglino, B.M. and Ravlin, E.C. (1998) "Individual values in organizations: Concepts, controversies, and research," Journal of management, vol. 24, pp. 351-389.

Otley, D. (1994) "Management control in contemporary organizations: towards a wider framework," Management accounting research, vol. 5, pp. 289-299.

Pavlov, I.P. (1941) "Lectures on conditioned reflexes. Vol. II. Conditioned reflexes and psychiatry,".

Place, U.T. (2000) "The role of the hand in the evolution of language," Psycoloquy, vol. 11.

Roediger III, H.L.; McDermott, K.B. and Robinson, K.J. (1998) "The role of associative processes in creating false memories," Theories of memory II, pp. 187-245.

Sitkin, S.B.; See, K.E.; Miller, C.C.; Lawless, M.W.; and Carton, A.M. (2011) "The paradox of stretch goals: Organizations in pursuit of the seemingly impossible," Academy of Management Review, vol. 36, pp. 544-566.

Skinner, B. F. (2014) Contingencies of reinforcement: A theoretical analysis vol. 3: BF Skinner Foundation.

Sober, E. (2014) "Mentalism and behaviorism in comparative psychology," in Comparing behavior, ed: Psychology Press, pp. 127-156.

Tolman, E.C. (1951) Purposive behavior in animals and men: Univ of California Press.

Watson, J. (1920) "Is thinking merely the action of language mechanisms? British journal of Psychology 11: 87104.[CP](1924) Behaviorism," ed: Norton [CP].

Wermers, R. (2003) "Is money really'smart'? New evidence on the relation between mutual fund flows, manager behavior, and performance persistence,".

$$
--0-
$$


